\title{
Placental Pathology Findings during and after SARS-CoV-2 Infection: Features of Villitis and Malperfusion
}

\author{
Thomas Menter ${ }^{\mathrm{a}} \quad$ Kirsten Diana Mertz $^{\mathrm{b}}$ Sizun Jiang ${ }^{\mathrm{c}}$ Han Chen $^{\mathrm{c}}$ \\ Cécile Monod $^{d}$ Alexandar Tzankov $^{\text {a Salome Waldvogel }}{ }^{\mathrm{e}}$ Sven M. Schulzke \\ Irene Höslid Elisabeth Bruder ${ }^{\mathrm{a}}$ \\ a Pathology, Institute of Medical Genetics and Pathology, University Hospital Basel, University of Basel, \\ Basel, Switzerland; ${ }^{\mathrm{b}}$ Institute of Pathology, Cantonal Hospital Baselland, Liestal, Switzerland; ${ }^{\mathrm{C}}$ Department of \\ Pathology, Stanford University, Stanford, CA, USA; ${ }^{\mathrm{d}}$ Department of Obstetrics and Antenatal Care, University \\ Hospital Basel, University of Basel, Basel, Switzerland; 'Department of Neonatology, University Children's Hospital \\ Basel UKBB, Basel, Switzerland
}

\section{Keywords}

Placenta · Malperfusion · COVID-19 · Chronic villitis

\begin{abstract}
Since the outbreak of coronavirus disease 2019 (COVID-19), there has been a debate whether pregnant women are at a specific risk for COVID-19 and whether it might be vertically transmittable through the placenta. We present a series of five placentas of SARS coronavirus 2 (SARS-CoV-2)-positive women who had been diagnosed with mild symptoms of COVID-19 or had been asymptomatic before birth. We provide a detailed histopathologic description of morphological changes accompanied by an analysis of presence of SARSCoV-2 in the placental tissue. All placentas were term deliveries (40th and 41 st gestational weeks). One SARS-CoV-2-positive patient presented with cough and dyspnoea. This placenta showed prominent lymphohistiocytic villitis and intervillositis and signs of maternal and foetal malperfusion. Viral RNA was present in both placenta tissue and the umbilical cord and could be visualized by in situ hybridization in the decidua. SARS-CoV-2 tests were negative at the time of
\end{abstract}

delivery of 3/5 women, and their placentas did not show increased inflammatory infiltrates. Signs of maternal and/or foetal malperfusion were present in $100 \%$ and $40 \%$ of cases, respectively. There was no transplacental transmission to the infants. In our cohort, we can document different time points regarding SARS-CoV-2 infection. In acute COVID-19, prominent lymphohistiocytic villitis may occur and might potentially be attributable to SARS-CoV-2 infection of the placenta. Furthermore, there are histopathological signs of maternal and foetal malperfusion, which might have a relationship to an altered coagulative or microangiopathic state induced by SARS-CoV-2, yet this cannot be proven considering a plethora of confounding factors.

() 2020 S. Karger AG, Basel

\section{Introduction}

Coronavirus disease 2019 (COVID-19) caused by SARS coronavirus 2 (SARS-CoV-2) has evolved into a worldwide pandemic within few months since its first documented appearance. Although severe courses and karger@karger.com

www.karger.com/pat

Karger ${ }^{\prime}=$
(C) 2020 S. Karger AG, Basel

Schönbeinstrasse 40

Thomas.Menter@usb.ch or Elisabeth.Bruder@usb.ch 
fatalities are primarily seen in elderly patients with relevant comorbidities, there are also younger patients showing adverse disease outcome $[1,2]$. We cannot know whether pregnant women are at higher risk of developing more severe complications than the general population as the absence of comparisons with appropriate controls is still lacking. Several studies like the national cohort studies from France or the UK concluded that the severity of COVID - 19 in pregnant women depends on comorbidities like age over 35 , body mass index (BMI) over 35, gestational diabetes, and hypertension $[3,4]$. Since its outbreak, there has also been a debate whether the disease as other viral infections - might influence foetal growth and be vertically transmittable through the placenta $[5$, 6].

The placenta is an immunoprivileged organ with attenuated immune response and a target of several viral infections [7]. Viruses such as cytomegaly virus (CMV), herpes simplex virus 1 and 2 (HSV), Rubella virus, human immunodeficiency virus (HIV), and lately also Zika virus have been shown to be able to cross the placental barrier and may be associated with severe foetal malformations $[8,9]$. The morphological response to viral infections varies: while CMV and HSV classically induce chronic lymphoplasmacytic villitis, in Zika virus infection, there is no inflammatory response, but a proliferation of Hofbauer cells (specialised placental macrophages) can be observed [10].

Pregnant women infected by other coronaviruses such as severe acute respiratory syndrome (SARS) and Middle East respiratory syndrome (MERS) have already been investigated in small case series. The rates of ICU admission and maternal death were significantly higher for pregnant women infected by SARS than in the general population and independent of the trimester of infection. Foetal outcome was characterized by a higher rate of miscarriage, intrauterine foetal death, and preterm deliveries [11]. Placental weight was below the 5 th percentile in a series of seven patients, of which two had abnormal pathology results (thrombotic vasculopathy with avascular fibrotic villi and/or placental infarct) [12]. When infection occurred during the week before birth, no foetal growth restriction was noted. When the infection occurred 1 month or more before birth, two foetuses $(2 / 3,33 \%)$ had foetal growth restriction with oligohydramnios, related to the abnormal placentas presented above. In the group of patients delivering during the acute phase of illness, placentas showed pronounced features of foetal and maternal malperfusion, but no increased inflammatory infiltrates or features of villitis were seen. Pooled information on pregnancy outcome in women infected by MERS did not show a risk for miscarriage, but a higher risk for preterm delivery and preeclampsia. None of the studies dealing with SARS or MERS showed signs of vertical transmission during the follow-up period [13]. So far, no morphologic evaluations of placentas of MERS-positive patients have been published [14, 15].

Several studies have already analysed the histomorphology of placentas in COVID-19 and could describe primarily microvascular changes, while an inflammatory response was rarely encountered [16-18]. Not all studies tested the placental tissue for the presence of the virus or, if so, have not morphologically analysed the tissue; therefore, it is still difficult to present a comprehensive overview of the interaction between SARS-CoV-2, COVID19-related complications, and the placenta. Furthermore, little is known about the time course of SARS-CoV-2 infection and morphologic alterations of the placenta.

Here we present a case series of five placentas of SARSCoV-2-positive women who had been diagnosed with virus infection before birth or at the day of birth. We provide a detailed histopathologic description of morphological changes accompanied by an analysis of inflammatory infiltrates, presence of SARS-CoV-2 RNA in placental tissue, and expression of the angiotensinconverting enzyme 2 (ACE2).

\section{Materials and Methods}

We included three women who had been tested antenatally for SARS-CoV-2 by nasal swabs because of symptoms and two asymptomatic pregnant women detected by universal screening when entering for delivery at the University Women's Hospital Basel, Switzerland since March 2020. The lockdown was imposed on March 15, 2020 in Switzerland. Altogether, five respective placenta specimens have since been sent for histologic examination to our institute.

After fixation for at least $48 \mathrm{~h}$ to reduce infectiousness, placentas were processed according to standard procedures, which include histologic examination of one block of the umbilical cord, one block of the chorionic membranes as well as three blocks of placenta tissue [19]. Additionally, macroscopically evident changes such as infarctions of hematomas were embedded. All slides were stained by haematoxylin and eosin. Pathological findings were classified according to the current Amsterdam Placental Workshop Group Consensus Statement [20].

In addition, one representative block of each placenta as well as of control placentas (5 cases with presence of lymphohistiocytic villitis and five cases without evidence of inflammatory infiltrates before the outbreak of COVID-19) were stained for fibrin (Dako, Glostrup, Denmark, A0080). Furthermore, both, the cohort of SARS-CoV-2 placentas and the control cohort, were stained for ACE2 (polyclonal antibody, Abcam ab15348, Cambridge, UK). 
Table 1. Clinical findings

\begin{tabular}{|c|c|c|c|c|c|}
\hline & Patient 1 & Patient 2 & Patient 3 & Patient 4 & Patient 5 \\
\hline Age at birth, years & 35.0 & 39.4 & 30.3 & 39.7 & 27.6 \\
\hline Type of birth & CS & CS & VD & VD & VD \\
\hline Gravida/para & G4P3 & G3P1 & G1P1 & G1P1 & G1P1 \\
\hline SARS-CoV-2 test positive prior to birth, days & 5 & 4 & 18 & -1 & 35 \\
\hline Gestational week of SARS-CoV-2 infection & $38+2$ & $38+3$ & $37+2$ & $40+5$ & $35+5$ \\
\hline SARS-CoV-2 test negative prior to birth, days & $\begin{array}{l}\text { Negative at the } \\
\text { day of delivery }\end{array}$ & $\begin{array}{l}\text { Negative } 25 \text { days } \\
\text { after delivery }\end{array}$ & 4 & $\begin{array}{l}\text { Negative } 3 \text { days } \\
\text { after delivery }\end{array}$ & 18 \\
\hline COVID-19-related symptoms & $\begin{array}{l}\text { None, detected } \\
\text { by screening }\end{array}$ & $\begin{array}{l}\text { Cough, } \\
\text { dyspnoea }\end{array}$ & Mild cough & $\begin{array}{l}\text { None, detected } \\
\text { by screening }\end{array}$ & Mild cough \\
\hline TORCH serology & Negative & Negative & Negative & Negative & Negative \\
\hline $\begin{array}{l}\text { Characteristics of neonates } \\
\text { Weight, } \mathrm{g}(\mathrm{Pc})\end{array}$ & $3,270(43)$ & $3,270(43)$ & $3,180(17)$ & $2,790(4)$ & $3,500(32)$ \\
\hline Length, $\mathrm{cm}(\mathrm{Pc})$ & $48.0(8)$ & $49.0(17)$ & $50.0(15)$ & $51.0(33)$ & $51.0(20)$ \\
\hline Head circumference, $\mathrm{cm}(\mathrm{Pc})$ & $35.0(62)$ & $34.0(32)$ & $33.5(6)$ & $33.0(6)$ & $35.0(26)$ \\
\hline Apgar score & $9 / 10 / 10$ & $9 / 10 / 10$ & $9 / 10 / 10$ & $8 / 9 / 9$ & $9 / 10 / 10$ \\
\hline Clinical course & NAD & $\begin{array}{l}\text { Transient hypo- } \\
\text { thermia }\left(35.9^{\circ} \mathrm{C}\right)\end{array}$ & NAD & NAD & NAD \\
\hline
\end{tabular}

BM, breast milk; CS, caesarean section; FM, formula milk; HELLP, haemolysis, elevated liver enzymes, low platelet count syndrome; NAD, no abnormalities detected in general appearance, heart rate, respiratory rate, or body temperature; Pc, percentile; VD, vaginal delivery.

A quantitative reverse transcription polymerase chain reaction (RT-qPCR) assay to detect the presence of SARS-CoV-2 RNA was performed on all samples as previously described [21]. In order to verify also the presence of SARS-CoV-2 RNA in foetal tissue, the umbilical cord was examined in addition to placental parenchyma.

For in situ hybridization, heat-induced epitope retrieval (HIER) was performed in a Lab Vision ${ }^{\mathrm{TM}} \mathrm{PT}$ module (Thermo Fisher) with Dako Target Retrieval Solution, pH 9 (S236784-2, DAKO Agilent, Santa Clara, CA, USA), for $10 \mathrm{~min}$ at $97^{\circ} \mathrm{C}$ before cooling down to $65^{\circ} \mathrm{C}$ and left to reach room temperature. Slides were then washed twice with Milli-Q water (Millipore Sigma, Burlington, MA, USA) before a 10 -min protease digestion at $40^{\circ} \mathrm{C}$ with 1:20 diluted Protease III (322337, Bio-Techne, Minneapolis, MN, USA) in $1 \times$ PBS. Slides were then washed for $2 \times 2$ min with Milli-Q water before a 15 -min block at $40^{\circ} \mathrm{C}(322335$, Bio-Techne). Slides were then washed for $2 \times 2$ min with Milli-Q water before an overnight hybridization at $40^{\circ} \mathrm{C}$ with probes against SARS-CoV-2 Spike mRNA (V-nCoV2019-S-sense, 848561, Bio-Techne). Amplification of the
ISH probes was performed the next day according to manufacturer's protocol (322350, Bio-Techne). Presence of viral RNA could be confirmed using a second probe (845701 RNAscope probe - V-nCoV2019-S-sense, Advanced Cell Diagnostics, Hayward, CA, USA). This staining was performed on the Leica Biosystems BOND III autostainer (Muttenz, Switzerland) according to the manufacturer's protocol.

\section{Results}

All placentas were term deliveries (40th and 41st gestational week). The mothers' age ranged between 27 and 39 years and the BMI between 26 and 35 at the time of birth. One patient (patient 2) presented with persistent cough and dyspnoea and was diagnosed with COVID-19 infection 4 days before delivery. We had to perform the 
caesarean section due to spontaneous rupture of membranes and transverse lie. The other pregnant women were all asymptomatic at the time of delivery. We could postpone one repeated caesarean section in a SARSCoV-2 positive, asymptomatic woman, detected by universal screening (patient 1). The positive swab result of the other pregnant woman screened for SARS-CoV-2 was obtained after delivery (patient 4). SARS-CoV-2 tests were negative at the time of delivery in $3 / 5$ women. All tests for SARS-CoV-2 of umbilical cord blood, breast milk, and amniotic fluid were negative.

Clinical findings of all patients and their neonates are summarized in Table 1. Histomorphological findings are summarized in Table 2 . No patient had a previous medical history of immunodeficiency or autoimmune disease.

The placenta of patient 1 (SARS-CoV-2 swab negative at the day of delivery) showed signs of maternal (increase of intervillous fibrin, decidual vasculopathy) and foetal

Table 2. Histomorphological findings

\begin{tabular}{|c|c|c|c|c|c|c|}
\hline & Patient 1 & Patient 2 & Patient 3 & Patient 4 & Patient 5 & Proportion \\
\hline Placental weight, g & 526 & 649 & 549 & 598 & 544 & n.a. \\
\hline Percentile & 60 & $>90$ & 60 & 65 & 60 & n.a. \\
\hline Neonatal weight, $g$ & 3,270 & 3,270 & 3,180 & 2,790 & 3,500 & \\
\hline Percentile & 40 & 45 & 35 & $<4$ & 40 & n.a. \\
\hline Ratio placental/foetal weight & 0.16 & 0.20 & 0.17 & 0.21 & 0.16 & n.a. \\
\hline Signs of maternal malperfusion & & & & & & $5 / 5$ \\
\hline Infarction & - & + & + & - & - & $2 / 5$ \\
\hline Increased perivillous fibrin deposition & - & - & + & - & + & $2 / 5$ \\
\hline Accelerated villous maturation & - & - & - & - & - & $0 / 5$ \\
\hline Tenney-Parker change & - & - & - & + & - & $1 / 5$ \\
\hline Decidual vasculopathy & + & & + & - & + & $3 / 5$ \\
\hline Increase of intervillous fibrin & + & + & + & - & + & $4 / 5$ \\
\hline Retroplacental haemorrhage & - & - & - & - & - & $0 / 5$ \\
\hline Intervillous thrombosis & - & + & - & - & - & $1 / 5$ \\
\hline Signs of foetal malperfusion & & & & & & $2 / 5$ \\
\hline Thrombi in the foetal circulation & + & + & - & - & - & $2 / 5$ \\
\hline Avascular villi & - & - & - & - & - & $0 / 5$ \\
\hline Karyorrhexis & - & - & - & - & - & $0 / 5$ \\
\hline Delayed villous maturation & + & - & - & - & - & $1 / 5$ \\
\hline Chorangiosis & + & + & - & - & - & $2 / 5$ \\
\hline \multicolumn{7}{|l|}{ Inflammatory changes } \\
\hline Chorioamnionitis & - & - & - & - & + & $1 / 5$ \\
\hline Chronic villitis & - & + & - & - & + & $2 / 5$ \\
\hline Chronic deciduitis & + & + & - & - & - & $2 / 5$ \\
\hline Subchorionitis & + & + & - & - & - & $2 / 5$ \\
\hline Choriovasculitis & - & + & - & - & - & $2 / 5$ \\
\hline Foetal vasculitis & - & + & - & - & - & $1 / 5$ \\
\hline Placenta accreta & - & + & - & - & - & $1 / 5$ \\
\hline Marginal insertion of the umbilical cord & + & - & - & - & - & $1 / 5$ \\
\hline Hypercoiling of the umbilical cord & + & - & + & + & - & $3 / 5$ \\
\hline Phagocytosis of meconium & - & + & - & - & - & $1 / 5$ \\
\hline Diffuse villous oedema & - & - & - & - & - & 0 \\
\hline
\end{tabular}

Fig. 1. Findings of the placenta with manifest COVID-19. a Macroscopic image showing inhomogeneous and unusually condensed placental parenchyma and an area of infarction (arrow). b Chronic villitis and intervillositis (haematoxylin and eosin [H\&E], 40×). c, d Characterisation of the inflammatory infiltrate consisting primarily of cytotoxic T-cells expressing CD8 (c) and fewer macrophages expressing CD68 (d) (immunohistochemistry, $200 \times$ ). e, f Lymphohistiocytic villitis resulting in chorionic vasculitis and subsequent fresh (e) and already organizing thrombosis (f) $(\mathrm{H} \& \mathrm{E}, 100 \times)$. g Intervillous increase of fibrin as result of maternal malperfusion $(\mathrm{H} \& \mathrm{E}, 100 \times)$. $\mathbf{h}$ Presence of SARS-CoV-2 in decidual cells (red) (in situ hybridization for SARS-CoV-2, 200×). 

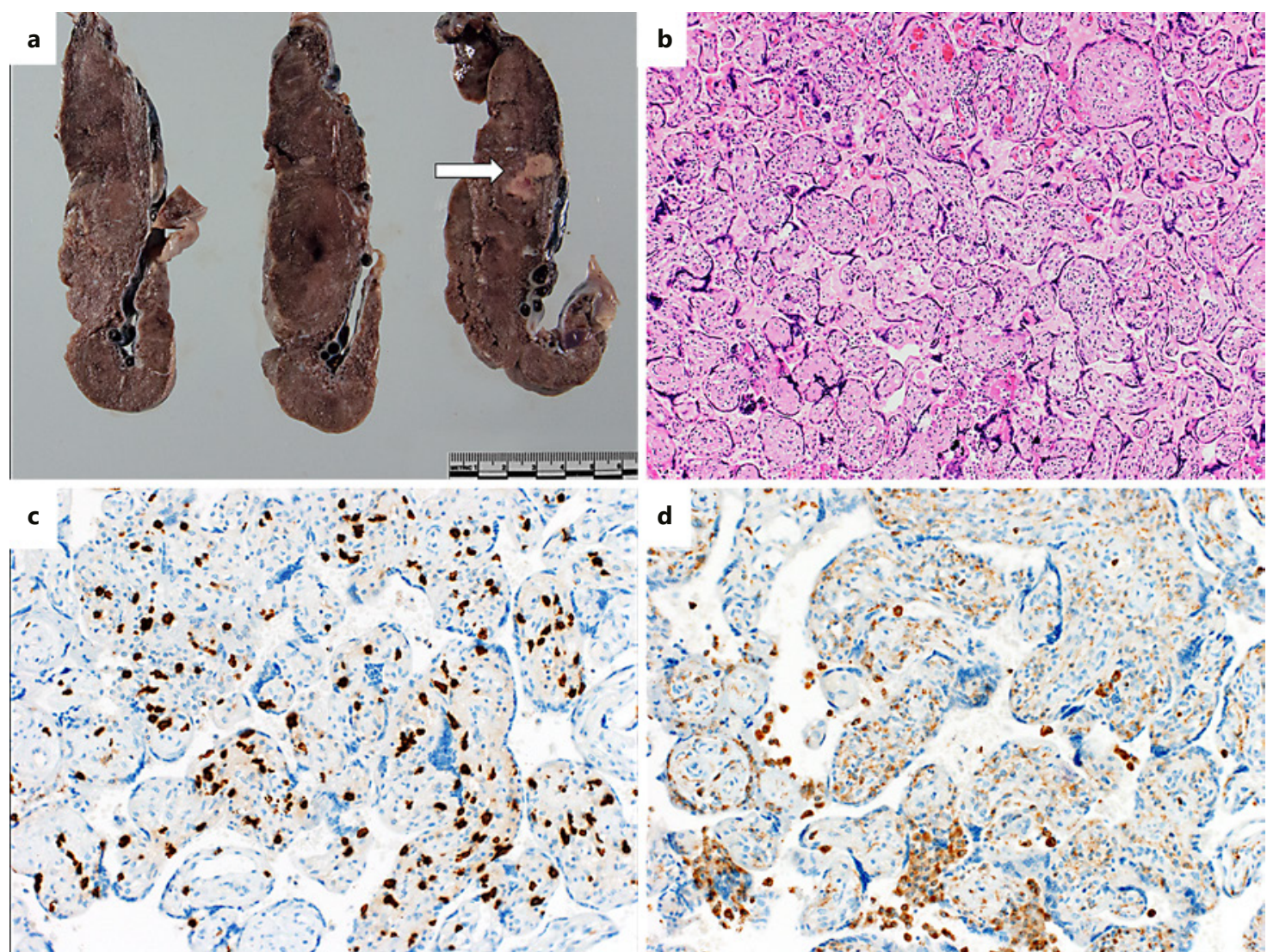

d $19-4 y^{2}$
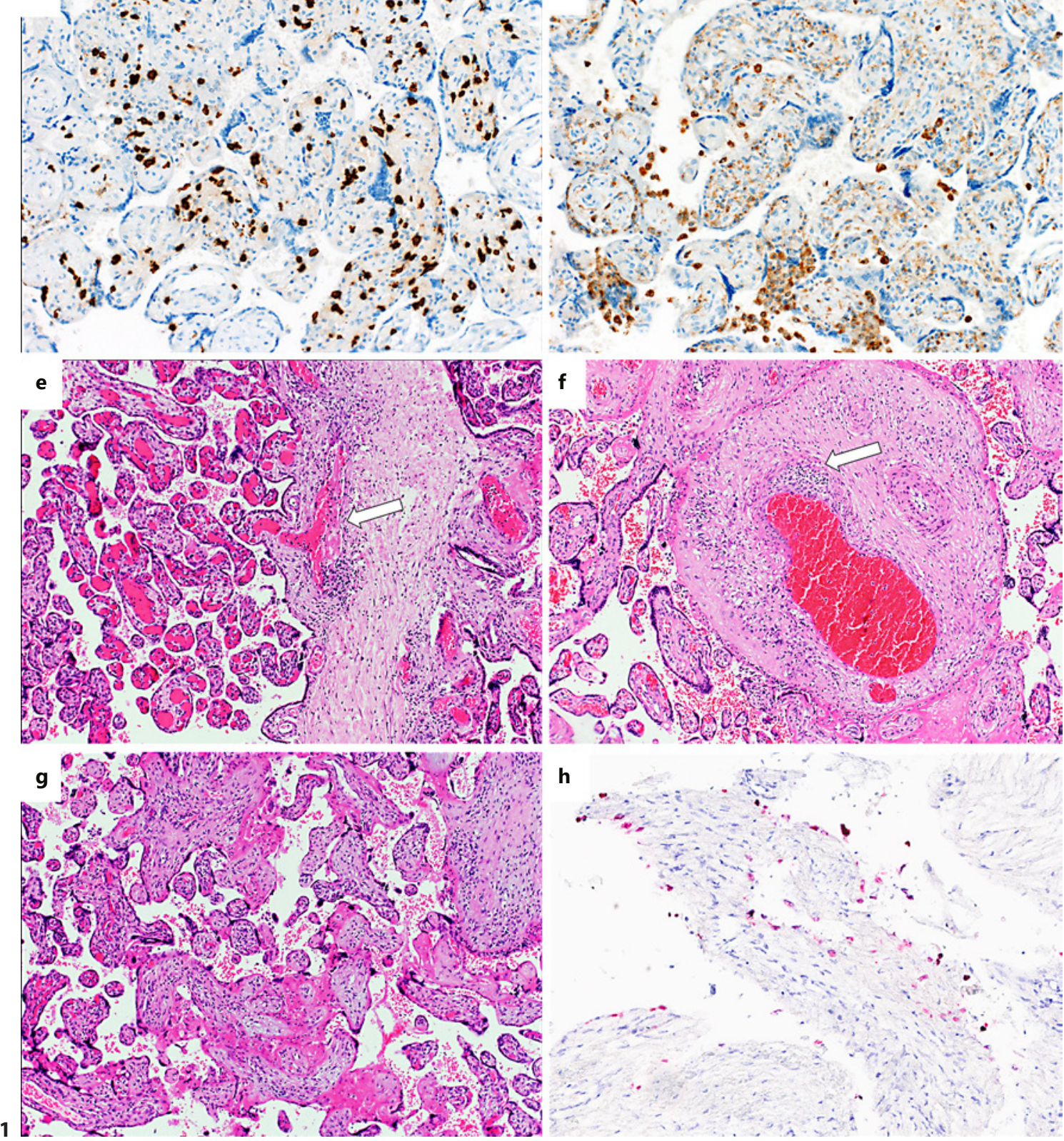
malperfusion (small thrombi in foetal vessels, delayed villous maturation, and chorangiosis) as well as mild chronic deciduitis and subchorionitis. There was also a marginal insertion of the umbilical cord and hypercoiling of the umbilical cord.

The placenta of patient 2 , who presented with COVID19-related symptoms at the time of delivery, showed a prominent lymphohistiocytic inflammatory infiltrate resulting in chronic lymphohistiocytic villitis and intervillositis accompanied by vasculitis of foetal vessels and focal thrombosis. The inflammatory infiltrate was predominantly composed of CD8-positive cytotoxic T-cells accompanied by mildly increased CD68-positive macrophages and scarce CD4-positive T-cells and plasma cells. There was no increase in neutrophils. The placenta furthermore showed signs of maternal (infarctions occupying $20 \%$ of the placental parenchyma, increase of intervillous fibrin, intervillous thrombosis) and foetal malperfusion (thrombi in chorionic vessels with vasculitis, delayed villous maturation, and chorangiosis). There was also focal amnial phagocytosis of meconium and features of placenta accreta. SARS-CoV-2 RNA was detectable at very low levels; however, it could be verified independently in different tissue blocks of umbilical cord and placental parenchyma. Furthermore, presence of SARS-CoV-2 was shown in the decidua by in situ hybridization. A nasal swab of the neonate was negative for SARS-CoV-2. A swab of the placental surface for SARS-CoV-2 was positive at the time of birth. Representative images of the placental findings are shown in Figure 1.

The placenta of patient 3 (SARS-CoV-2 swab negative 4 days prior to delivery) showed signs of maternal malperfusion (infarction occupying $10 \%$ of the placental parenchyma, increase of intervillous fibrin, perivillous fibrin deposition [gitterinfarcts], decidual vasculopathy) and hypercoiling of the umbilical cord. There were no increased inflammatory infiltrates. This patient was a smoker during pregnancy and had had bariatric surgery.

The placenta of patient 4 (SARS-CoV-2 swab positive at the day of delivery) also showed signs of maternal malperfusion (Tenney-Parker changes) and hypercoiling of the umbilical cord. As in patient 3, there were no increased inflammatory infiltrates. Foetal thoracic cystic malformation had been suspected at 22 weeks of gestation and several weeks before the COVID-19 infection. Postnatally, chest X-ray and thoracic ultrasound studies as well as echocardiography and cranial ultrasound of this child were unremarkable.

The placenta of patient 5 (SARS-CoV-2 swabs persistently negative for 18 days prior to delivery) showed flor- id chorioamnionitis as well as focal chronic villitis (much less extensive than patient 2). No microbiological investigation for the analysis of the speculated ascending infection was performed. There was also mild vasculitis of the decidua. In addition, it showed signs of maternal malperfusion (increase of intervillous fibrin, perivillous fibrin deposition [gitterinfarcts], decidual vasculopathy).

Findings of the four patients without COVID-19 symptoms at the time of delivery are shown in Figure $2 \mathrm{a}-\mathrm{c}$.

Fibrin staining showed a small thrombus in a foetal vessel of patient 2. All other placentas of the SARASCoV-2 cohort and the control group did not show thrombi in foetal vessels. Analysis of expression of ACE2 showed weak expression in cells of the invasive extravillous trophoblast in $4 / 5$ cases of our cohort as well as $8 / 10$ control cases (Fig. 2d). In decidual stroma cells and decidualized endometrial glands, focal very weak ACE2 expression was noted.

\section{Discussion}

In accordance with other studies, we could show that microvasculopathy, which manifests as signs of maternal malperfusion and to a lesser degree as foetal malperfusion, is a common finding in placentas of SARS-CoV2-positive women occurring in all and 2/5 patients of our cohort, respectively. In our case of acute COVID-19 (patient 2), prominent lymphohistiocytic villitis occurred, which might be potentially attributable to SARS-CoV-2 infection of the placenta. We could document different time points in regard to SARS-CoV-2 infection ranging from clearance of the virus for several weeks to both acute symptomatic and asymptomatic infections. This feature has not been documented systematically in the literature yet. Furthermore, presence of SARS-CoV-2 RNA in placental tissue is still a rarity.

In our cohort, we could see that findings differ in relation to the time of detection and clearance of SARSCoV-2. Our case with manifest COVID-19 disease showed the most prominent inflammatory response as well as presence of viral RNA in both the placenta and the umbilical cord and presence of SARS-CoV-2 in the decidua. These findings support the hypothesis that SARSCoV-2 can invade the placenta, cause an inflammatory response, and is potentially transmittable to the child. They also corroborate findings of Patanè et al. [6] and other studies looking at the presence of the virus or antiSARS-CoV-2 antibodies in neonates [22-24]. In contrast
Menter et al. 


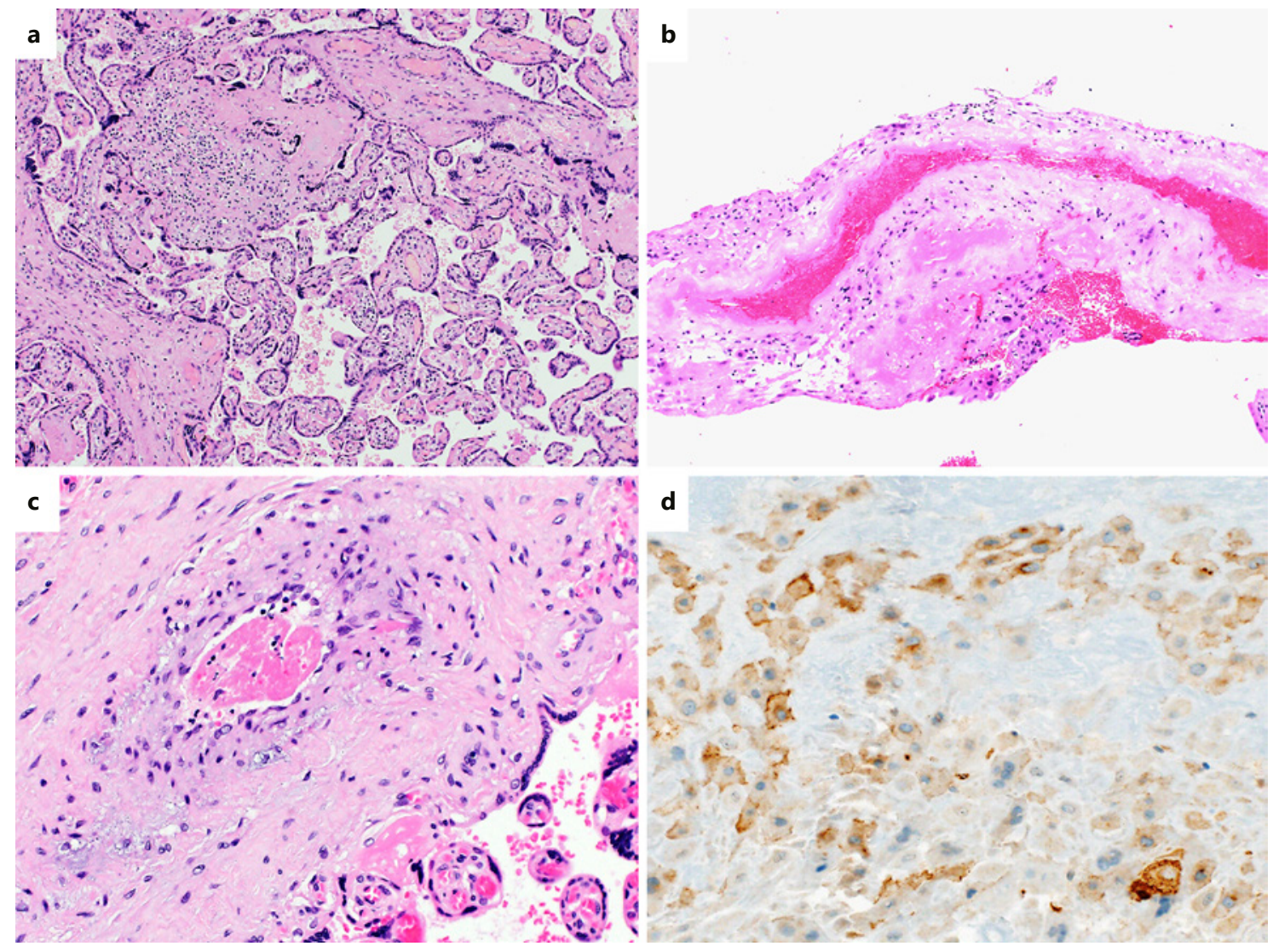

Fig. 2. Findings of placentas with no presence of SARS-CoV-2 at the time of delivery and expression of ACE2. a Features of subtle chronic villitis in patient 5 showing acute chorioamnionitis as major finding. The lymphohistiocytic infiltrate was sparse in contrast to patient $2(\mathrm{H} \& \mathrm{E}, 100 \times)$. b Decidual arteriopathy in patient 1 : decidual artery showing complete necrosis of the arterial wall and intraluminal fibrosis. The patient did not show evidence of pre- eclampsia or gestation-related hypertension. c Foetal vessel in patient 1 showing a small thrombus. In contrast to patient 2, the thrombus was not wall-adherent and there were no signs of vasculitis (H\&E, 200×). c, d Immunohistochemistry for ACE2 showing weak expression in the extravillous invasive trophoblast (immunohistochemistry, $40 \times)$. to these case reports, intervillositis was not as pronounced; however, we could document foci of vasculitis. Characterizing the cellular composition of the inflammatory infiltrate, it did not differ from cases of CMV placentitis (except for lack of increase of plasma cells) or chronic villitis of unknown aetiology. Our patient also did not have a medical history of autoimmune diseases, which might be one of the causes of villitis of unknown aetiology. However, she suffered from insulin-dependent gestational diabetes and had a history of preeclampsia in her first pregnancy. She was under aspirin for prevention of preeclampsia until week 36. TORCH serology as well as immunohistochemical exams of the placenta tissue for infectious agents (adenovirus, CMV, HSV, toxoplasmosis) had been negative. Postnatal transition and clinical course of all five neonates were unremarkable apart from tran- sient hypothermia of one neonate (child to patient No. 2), which was considered to be due to environmental factors. All children received breast milk.

The largest series published so far, consisting of 20 placentas, reports chronic villitis in four patients, yet those did not seem to show symptoms of COVID-19 [17]. On the other hand, three patients with symptoms of COVID-19 did not show chronic villitis, with one patient showing signs of acute ascending infection. Shanes et al. [16] investigated 16 placentas of SARS-CoV-2-positive patients and compared their results to large historical pre-COVID-19 control groups. They could find chronic villitis in two cases, one of these patients was in need of oxygen. Compared to their control groups, chronic villitis in general was not more common in SARS-CoV-2-positive patients in contrast to signs of maternal malperfu- 
sion. Unfortunately, the authors of these studies did not report whether the women were tested again at the time of delivery after SARS-CoV-2 had been detected earlier in pregnancy. The placental tissue had not been tested for presence of SARS-CoV-2 RNA in these studies.

SARS-CoV-2 has an impact on coagulation, yet coagulation is already altered by pregnancy itself [25]. Whether SARS-CoV-2 can be attributable to the vascular and circulatory pathologies still needs further studies. It is important to distinguish between acute and chronic changes of the placenta as features such as infarctions, delayed villous maturation, avascular fibrosed villi, or chorangiosis need time to evolve. On the other hand, the patients of our cohort did not have pregnancy-related hypertension, which is - in contrast to obesity and gestational diabetes mellitus - a well-established risk factor for maternal malperfusion of the placenta [26]. In the only available cohort of seven SARS-patients, those having had SARS earlier in pregnancy showed normal placental histology [12]. Our patient with manifest COVID-19 showed no abnormalities of her coagulation parameters and there was no documentation of thromboembolic events. Three weeks after delivery, the patient suffered from an episode of acute cholecystitis due to cholecystolithiasis and the gall bladder was removed. This specimen also did not show evidence of current or organized thrombosis. The foetal thrombi were associated with those vessels showing vasculitis, so here the link of thrombi and infection/inflammation is more likely. Mulvey et al. [18] investigated five cases of the largest series published with signs of foetal malperfusion for activation of the complement pathway, yet these investigations turned out negative. Additionally, these five cases did not show evidence of villitis. In three of our cases, the diagnosis of presence of SARS-CoV-2 was made less than 1 week prior to delivery and less than 4 weeks since the first confirmed case in Switzerland in two of these cases. The longest interval of our cohort between detection of SARS-CoV-2 and delivery was 5 weeks and nasal swabs had been consistently negative for 3 weeks prior to delivery. This patient showed discrete decidual vasculopathy and only mild increase of intervillous fibrin. There was also discrete focal chronic villitis; however, this was much less pronounced than in patient 2 having manifest COVID-19.

In accordance with previous studies on ACE2 [27, 28], there was only weak immunohistochemical expression of ACE2 in the invasive extravillous trophoblast of both our SARS-CoV-2 cohort and control cases. Expression of ACE2 in other cell types was scarce to absent. SARSCoV-2 RNA was exclusively seen in transformed epithe- lial cells of the decidua, which showed very week expression of ACE2. Studies on animal models have described weak expression of ACE2 in endometrial cells of pregnant rats [29]. Expression of ACE2 in human endometrial cells in pregnancy is not described yet; however, first reports on ACE2 expression in the endometrium are currently being published [30, 31].

To conclude, our study shows the variegated spectrum of findings in women infected with SARS-CoV-2. Caution has to be taken to consider pregnant women as a homogenous group as outcomes might depend on the current state of the infection considering manifest COVID-19 as well as a state after which virus clearance has already been achieved. We could show that in acute COVID-19, prominent lymphohistiocytic villitis may occur and might be attributable to SARS-CoV-2 infection of the placenta. Furthermore, there are pathological findings of maternal and foetal malperfusion which might have a relationship to an altered coagulative state induced by SARS-CoV-2, yet this cannot be consistently proven considering the plethora of confounding factors.

\section{Statement of Ethics}

This study has received permission by the local ethics committee of central and Northwestern Switzerland (EKNZ, ID 202000629).

\section{Conflict of Interest Statement}

The authors declare to have no competing interests.

\section{Funding Sources}

This work is supported by the Botnar Research Centre for Child Health, BRCCH. S.J. is supported by the Leukemia \& Lymphoma Society Career Development Program, a Fast Grant Funding for COVID-19 Science and the Bill \& Melinda Gates Foundation OPP1113682.

\section{Author Contributions}

T.M., I.H., and E.B. designed the study. T.M. wrote the manuscript. K.D.M. performed qPCR analysis. S.J. and H.C. performed in situ hybridization for SARS-CoV-2. A.T. analysed expression of ACE2. S.W., S.M.S., C.M., and I.H. provided clinical data. All authors critically read and approved the manuscript.
Menter et al. 


\section{References}

1 Petrilli CM, Jones SA, Yang J, Rajagopalan H, O’Donnell L, Chernyak Y, et al. Factors associated with hospital admission and critical illness among 5279 people with coronavirus disease 2019 in New York City: prospective cohort study. BMJ. 2020 May;369:m1966.

2 Docherty AB, Harrison EM, Green CA, Hardwick HE, Pius R, Norman L, et al.; ISARIC4C investigators. Features of 20133 UK patients in hospital with covid-19 using the ISARIC WHO Clinical Characterisation Protocol: prospective observational cohort study. BMJ. 2020 May;369:m1985.

3 Kayem G, Lecarpentier E, Deruelle P, Bretelle F, Azria E, Blanc J, et al. A snapshot of the Covid-19 pandemic among pregnant women in France. J Gynecol Obstet Hum Reprod. 2020 Sep;49(7):101826.

4 Knight M, Bunch K, Vousden N, Morris E, Simpson N, Gale C, et al.; UK Obstetric Surveillance System SARS-CoV-2 Infection in Pregnancy Collaborative Group. Characteristics and outcomes of pregnant women admitted to hospital with confirmed SARS-CoV-2 infection in UK: national population based cohort study. BMJ. 2020 Jun;369:m2107.

5 Penfield CA, Brubaker SG, Limaye MA, Lighter J, Ratner AJ, Thomas KM, et al. Detection of SARS-COV-2 in Placental and Fetal Membrane Samples. Am J Obstet Gynecol MFM. 2020;2(3):100133.

6 Patanè L, Morotti D, Giunta MR, Sigismondi C, Piccoli MG, Frigerio L, et al. Vertical transmission of coronavirus disease 2019: severe acute respiratory syndrome coronavirus 2 RNA on the fetal side of the placenta in pregnancies with coronavirus disease 2019-positive mothers and neonates at birth. Am J Obstet Gynecol MFM. 2020 Aug;2(3): 100145.

7 Racicot K, Mor G. Risks associated with viral infections during pregnancy. J Clin Invest. 2017 May; 127(5):1591-9.

8 Lee JK, Oh SJ, Park H, Shin OS. Recent Updates on Research Models and Tools to Study Virus-Host Interactions at the Placenta. Viruses. 2019 Dec;12(1):E5.

9 Antoniou E, Orovou E, Sarella A, Iliadou M, Rigas N, Palaska E, et al. Zika Virus and the Risk of Developing Microcephaly in Infants: A Systematic Review. Int J Environ Res Public Health. 2020 May;17(11):E3806.

10 Schwartz DA. Viral infection, proliferation, and hyperplasia of Hofbauer cells and absence of inflammation characterize the placental pathology of fetuses with congenital Zika virus infection. Arch Gynecol Obstet. 2017 Jun; 295(6):1361-8.

11 Lambelet V, Vouga M, Pomar L, Favre G, Gerbier E, Panchaud A, et al. SARS-CoV-2 in the context of past coronaviruses epidemics: consideration for prenatal care. Prenat Diagn. 2020 May;pd.5759.

$12 \mathrm{Ng}$ WF, Wong SF, Lam A, Mak YF, Yao H, Lee $\mathrm{KC}$, et al. The placentas of patients with severe acute respiratory syndrome: a pathophysiological evaluation. Pathology. 2006 Jun;38(3): 210-8.

13 Di Mascio D, Khalil A, Saccone G, Rizzo G, Buca D, Liberati M, et al. Outcome of coronavirus spectrum infections (SARS, MERS, COVID-19) during pregnancy: a systematic review and meta-analysis. Am J Obstet Gynecol MFM. 2020 May;2(2):100107.

14 Schwartz DA, Graham AL. Potential Maternal and Infant Outcomes from (Wuhan) Coronavirus 2019-nCoV Infecting Pregnant Women: Lessons from SARS, MERS, and Other Human Coronavirus Infections. Viruses. 2020 Feb;12(2):E194.

15 Liu W, Wang J, Li W, Zhou Z, Liu S, Rong Z. Clinical characteristics of 19 neonates born to mothers with COVID-19. Front Med. 2020 Apr;14(2):193-8.

16 Shanes ED, Mithal LB, Otero S, Azad HA, Miller ES, Goldstein JA. Placental Pathology in COVID-19. Am J Clin Pathol. 2020 Jun; 154(1):23-32.

17 Baergen RN, Heller DS. Placental Pathology in Covid-19 Positive Mothers: preliminary Findings. Pediatr Dev Pathol. 2020 May-Jun; 23(3):177-80.

18 Mulvey JJ, Magro CM, Ma LX, Nuovo GJ, Baergen RN. Analysis of complement deposition and viral RNA in placentas of COVID-19 patients. Ann Diagn Pathol. 2020 Jun;46: 151530.

19 sgpath.ch/docs/QRL/QR_SGPath_DE_2011. pdf.

20 Khong TY, Mooney EE, Ariel I, Balmus NC, Boyd TK, Brundler MA, et al. Sampling and Definitions of Placental Lesions: Amsterdam Placental Workshop Group Consensus Statement. Arch Pathol Lab Med. 2016 Jul;140(7): 698-713.

21 Menter T, Haslbauer JD, Nienhold R, Savic S, Hopfer H, Deigendesch N, et al. Postmortem examination of COVID-19 patients reveals diffuse alveolar damage with severe capillary congestion and variegated findings in lungs and other organs suggesting vascular dysfunction. Histopathology. 2020 May;77(2): 198-209.

22 Egloff C, Vauloup-Fellous C, Picone O, Mandelbrot L, Roques P. Evidence and possible mechanisms of rare maternal-fetal transmission of SARS-CoV-2. J Clin Virol. 2020 Jul; 128:104447.

23 Hosier H, Farhadian SF, Morotti RA, Deshmukh U, Lu-Culligan A, Campbell KH, et al. SARS-CoV-2 infection of the placenta. J Clin Invest. 2020 Sep;130(9):4947-53.

24 Vivanti AJ, Vauloup-Fellous C, Prevot S, Zupan V, Suffee C, Do Cao J, et al. Transplacental transmission of SARS-CoV-2 infection. Nat Commun. 2020 Jul;11(1):3572.

25 Benhamou D, Keita H, Ducloy-Bouthors AS; CARO working group. Coagulation changes and thromboembolic risk in COVID-19 obstetric patients. Anaesth Crit Care Pain Med 2020;39(3):351-353.

26 Bustamante Helfrich B, Chilukuri N, He H, Cerda SR, Hong X, Wang G, et al. Maternal vascular malperfusion of the placental bed associated with hypertensive disorders in the Boston Birth Cohort. Placenta. 2017 Apr;52: 106-13.

27 Pringle KG, Tadros MA, Callister RJ, Lumbers ER. The expression and localization of the human placental prorenin/renin-angiotensin system throughout pregnancy: roles in trophoblast invasion and angiogenesis? Placenta. 2011 Dec;32(12):956-62.

28 Valdés G, Neves LA, Anton L, Corthorn J, Chacón C, Germain AM, et al. Distribution of angiotensin-(1-7) and ACE2 in human placentas of normal and pathological pregnancies. Placenta. 2006 Feb-Mar;27(2-3):200-7.

29 Neves LA, Stovall K, Joyner J, Valdés G, Gallagher PE, Ferrario CM, et al. ACE2 and ANG-(1-7) in the rat uterus during early and late gestation. Am J Physiol Regul Integr Comp Physiol. 2008 Jan;294(1):R151-61.

30 Henarejos-Castillo I, Sebastian-Leon P, Devesa-Peiro A, Pellicer A, Diaz-Gimeno P. SARSCoV-2 infection risk assessment in the endometrium: viral infection-related gene expression across the menstrual cycle. Fertil Steril. 2020 Aug; 114(2):223-32.

31 Chadchan SB, Maurya VK, Popli P, Kommagani R. The SARS-CoV-2 receptor, Angiotensin converting enzyme 2 (ACE2) is required for human endometrial stromal cell decidualization. bioRxiv. 2020. https://doi. org/10.1101/2020.06.23.168252. 